\title{
ReVIEWer Acknowledgements 2021
}

Dear reviewers, on behalf of the editors of section and myself, we extend our sincere thanks for your commitment to Botanical Sciences in reviewing the manuscripts of this volume 99.

Thank you for your expertise and input.

Teresa Terrazas

\author{
Rafael Acuña Castillo \\ Universidad de Costa Rica, Costa Rica \\ Karla M. Aguilar Dorantes \\ Universidad Autónoma del Estado de México, México \\ Xitlali Aguirre Dugua \\ Colegio de Postgraduados, México \\ Antonio Andrade-Torres \\ Universidad Veracruzana, México \\ Agustina Rosa Andrés-Hernández \\ Benemérita Universidad Autónoma de Puebla, México \\ Guadalupe Ángeles López \\ Universidad Nacional Autónoma de México, México \\ José Luis Aragón-Gastélum \\ Universidad Autónoma de Campeche, México \\ Gabriel Arroyo-Cosultchi \\ Universidad Nacional Autónoma de México, México \\ José Guillermo Ávila Acevedo \\ Universidad Nacional Autónoma de México, México \\ Eleinis Ávila-Lovera \\ Universidad de California, Riverside, USA \\ Gloria Estela Barboza \\ Universidad Nacional de Córdoba, Argentina \\ Jorge Luis Becerra López \\ Universidad Juárez del Estado de Durango, México \\ Johannes M. Bouchal \\ Sewdish Museum, Sweden \\ Carlos Manuel Burelo-Ramos \\ Universidad Juárez Autónoma de Tabasco, México \\ Dánae Cabrera Toledo \\ Universidad de Guadalajara, México \\ Manuel Jesús Cach Pérez \\ Colegio de la Frontera Sur, México \\ Luz María Calvo Irabien \\ Centro de Investigación Cientifica de Yucatán AC. México \\ Fernando Calzada \\ Instituto Mexicano del Seguro Social, México
}

\author{
Ana Lorena Carrera \\ Consejo Nacional de Investigaciones Científicas y Técnicas de \\ Argentina, Argentina \\ María Guadalupe Carrillo Galván \\ Universidad Nacional Autónoma de México, México \\ Silvia Castillo Argüero \\ Universidad Nacional Autónoma de México, México \\ Gonzalo Castillo-Campos \\ Instituto de Ecología, AC. México \\ María Chávez Pesqueira \\ Centro de Investigación Científica de Yucatán, México \\ Bruno Manuel Chávez Vergara \\ Universidad Nacional Autónoma de México, México \\ Franco Ezequiel Chiarini \\ Museo Botánico de Córdoba, Argentina \\ Virginia Gabriela Cilia López \\ Universidad Autónoma de San Luis Potosí, México \\ Eliezer Cocoletzi \\ Instituto de Ecología AC, México \\ Patricia Colunga \\ Investigador Independiente, México \\ Raúl Contreras Medina \\ Universidad Autónoma Benito Juárez de Oaxaca, México \\ Allen Coombes \\ Benemérita Universidad Autónoma de Puebla, México \\ Guadalupe Cornejo-Tenorio \\ Universidad Nacional Autónoma de México, México \\ Jorge Cortés-Flores \\ Universidad Nacional Autónoma de México, México \\ Ek de Val de Gortari \\ Universidad Nacional Autónoma de México, México \\ Paulina Del Valle \\ Universidad Nacional Autónoma de México, México \\ Pablo Delgado Sánchez \\ Universidad Autónoma de San Luis Potosí, México
}

This is an open access article distributed under the terms of the Creative Commons Attribution License CCBY-NC (4.0) international. https://creativecommons.org/licenses/by-nc/4.0/ 
Rodolfo Dirzo

Universidad de Stanford, USA

David Douterlungne

Instituto Potosino de Investigación Cientifica y Tecnológica AC, México

Rodrigo Duno de Stefano

Centro de Investigación Científica de Yucatán, México Juan Manuel Dupuy-Rada

Centro de Investigación Cientifica de Yucatán, México

Francisca Ely

Universidad de los Andes, Venezuela

Juan Antonio Encina Domínguez

Universidad Autónoma Agraria Antonio Narro, México

Adrián José Enríquez Valencia

Instituto de Ecología AC, México

Tania Escalante-Espinosa

Universidad Nacional Autónoma de México, México Adolfo Espejo Serna

Universidad Autónoma Metropolitana, México

Silvia Espinosa Matías

Universidad Nacional Autónoma de México, México

David Nahum Espinosa Organista

Universidad Nacional Autónoma de México, México Eduardo Estrada

Universidad Autónoma de Nuevo León, México

Emilio Estrada Ruiz

Instituto Politécnico Nacional, México

Coyolxauhqui Figueroa Batalla

Universidad Autónoma de Ciudad Juárez, México

Dulce María Figueroa Castro

Benemérita Universidad Autónoma de Puebla, México

Mark Fishbein

Oklahoma State University, USA

José Germán Flores Garnica

Instituto Nacional de Investigaciones Forestales Agrícolas y

Pecuarias, México

Alejandro Flores-Palacios

Universidad Autónoma del Estado de México, México Itzi Fragoso Martínez

Universidad Nacional Autónoma de México, México Deneb García Ávila

Universidad Michoacana de San Nicolás de Hidalgo, México José G. García Franco

Instituto de Ecología, AC. México

Abisai García-Mendoza

Universidad Nacional Autónoma de México, México

René Garruña Hernández

Tecnológico Nacional de México, México Jordan Goluvob

Universidad Autónoma Metropolitana, México

Sandra Luz Gómez Acevedo

Universidad Nacional Autónoma de México, México
Maricela Gómez Sánchez

Universidad Autónoma de Querétaro, México

Dolores González

Instituto de Ecología AC, México

Fidel González Torralva

University of Arkansas, USA

Jesús Guadalupe González-Gallegos

Instituto Politécnico Nacional, México

Marina Gotelli

Universidad de Buenos Aires, Argentina

Gabriel Graciano Ávila

Universidad para el Bienestar Benito Juárez García, México Jessica Granados

Henry Ford Health System, USA

Jorge Alberto Gutiérrez

Universidad Autónoma de Chapingo, México

Adela Monserrat Gutiérrez Bustillo

Universidad Complutense de Madrid, España

Silvia Laura Guzmán Gutiérrez

Universidad Nacional Autónoma de México, México

Patricia Herrera Paniagua

Universidad Autónoma de Querétaro, México

Yolanda Herrera-Arrieta

Instituto Politécnico Nacional, México

Yvonne Herrerías-Diego

Universidad Michoacana de San Nicolás de Hidalgo, México Cecilia Jiménez

Universidad Autónoma Metropolitana, México

Maribel Jiménez Fernández

Universidad Veracruzana, México

Daniel Jiménez García

Benemérita Universidad Autónoma de Puebla, México

Ana Laura Lara Domínguez

Instituto de Ecología AC, México

Carlos Luis Leopardi Verde

Universidad de Colima, México Juan Carlos López Acosta

Universidad Veracruzana, México Mauricio Lopes de Faria

Universidade Estadual de Montes Claros, Brasil

Berenice Nataly López Gutiérrez

Universidad Autónoma del Estado de Hidalgo, México Cristina López Peralta

Colegio de Posgraduados, México

Jorge Manuel López-Calderón

Universidad Autónoma de Baja California, México

María del Socorro Lozano

Universidad Nacional Autónoma de México, México

Yurixhi Maldonado-López

Universidad Michoacana de San Nicolás de Hidalgo, México

Cristian Adrián Martínez Adriano

Universidad Autónoma de Nuevo León, México 


\author{
Concepción Martínez Peralta \\ Universidad Autónoma del Estado de Morelos, México \\ José Guadalupe Martínez-Ávalos \\ Universidad Autónoma de Tamaulipas, México \\ Yuriana Martínez-Orea \\ Universidad Nacional Autónoma de México, México \\ José Luis Martínez-Sánchez \\ Universidad Juárez Autónoma de Tabasco, México \\ Jorge Meave \\ Universidad Nacional Autónoma de México, México \\ Ernesto Medina \\ Instituto Venezolano de Investigaciones Cientificas (IVIC), \\ Venezuela \\ Rodrigo Méndez Alonzo \\ Centro de Investigación Cientifica y de Educación Superior de \\ Ensenada, México \\ Pedro Eloy Mendoza Hernández \\ Universidad Nacional Autónoma de México, México \\ Antonio Miranda \\ Universidad Veracruzana, México \\ Vivian Molina Cuevas \\ Centro Nacional de Investigaciones Científicas, Cuba \\ Francisco Molinar Holguín \\ Universidad Autónoma de Ciudad Juárez, México \\ Noé Manuel Montaño Arias \\ Universidad Autónoma Metropolitana, México \\ Eduardo A. Morales \\ Universidade de Évora, Portugal \\ Viridiana Morales Sánchez \\ Consejo Superior de Investigaciones Cientificas, España \\ Guadalupe Munguía Lino \\ Universidad de Guadalajara, México \\ Alethia Muñiz-Ramírez \\ Instituto Potosino de Investigación Cientifica y Tecnológica AC, \\ México \\ Rodrigo Muñoz Aviles \\ Wageningen University and Research, Netherlands \\ Daniela S. Nitiu \\ Universidad Nacional de la Plata, Argentina \\ Eberto Novelo Maldonado \\ Universidad Nacional Autónoma de México, México \\ Jonathan Ochoa-Gómez \\ Universidad Autónoma del Carmen, México \\ Denise Oliveira \\ Universidade Federal de Minas Gerais, Brasil \\ Marisela Pando-Morero \\ Universidad Autónoma de Nuevo León, México \\ José L. Panero \\ University of Texas at Austin, USA \\ Guillermo Pastor-Palacios \\ Instituto Potosino de Investigación Cientifica y Tecnológica AC, \\ México
}

Eduardo A. Pérez García

Universidad Nacional Autónoma de México, México Marlín Pérez Suárez

Universidad Autónoma del Estado de México, México Araceli Pérez Vásquez

Universidad Nacional Autónoma de México, México Liberato Portillo

Universidad de Guadalajara, México

Liliana Ramírez Freire

Universidad Autónoma de Nuevo León, México

Alfredo Ramírez Hernández

Instituto Potosino de Investigación Científica y Tecnológica AC, México

Neptalí Ramírez-Marcial

Colegio de la Frontera Sur, México

Rosario Redonda Martínez

Instituto de Ecología AC, México

Juan Antonio Reyes-Agüero

Universidad Autónoma de San Luis Potosí, México

José Rivera-Chávez

Universidad Nacional Autónoma de México, México

Hernando Rodríguez Correa

Universidad Nacional Autónoma de México, México

Nelson Facundo Rodríguez López

Universidad Industrial de Santander, Colombia

Mario Rodríguez-Monroy

Instituto Politécnico Nacional, México

Oris Rodríguez-Reyes

Universidad de Panamá, Panamá

Fernando Rosas-Pacheco

Universidad Autónoma del Estado de Hidalgo, México

Daniel Sánchez

Universidad de Guadalajara, México

Gerardo Sánchez Ramos

Universidad Autónoma de Tamaulipas, México

Arturo Sánchez-González

Universidad Autónoma del Estado de Hidalgo, México

Fernando Santacruz

Universidad de Guadalajara, México

Lislie Solís

Colegio de la Frontera Sur, México

Leticia Souto

Universidade Federal de São Carlos, Brasil

Víctor Werner Steinmann

Universidad Autónoma de Querétaro, México Carla Etel Suárez

Universidad Nacional de La Pampa, Argentina José Luis Tapia-Muñoz

Centro de Investigación Cientifica de Yucatán, México Ignacio Torres García

Universidad Nacional Autónoma de México, México 
Cesar Andrés Torres-Miranda

Universidad Nacional Autónoma de México, México Alejandro Torres-Montúfar

Universidad Nacional Autónoma de México, México Susana Valencia-Avalos

Universidad Nacional Autónoma de México, México Alfonso Valiente-Banuet

Universidad Nacional Autónoma de México, México Carlos Fabián Vargas Mendoza

Instituto Politécnico Nacional, México

Marco Antonio Vásquez-Dávila

Instituto Tecnológico del Valle de Oaxaca, México Sonia Vázquez-Santana

Universidad Nacional Autónoma de México, México Noé Velázquez-Rosas

Universidad Veracruzana, México
Pedro Viana

Museu Paraense Emílio Goeldi, Brasil

Heike Vibrans

Colegio de Posgraduados, México José A. Villarreal

Universidad Autónoma Agraria Antonio Narro, México Fernando Vite

Universidad Autónoma Metropolitana, México Israel Yerena

Universidad Autónoma de Nuevo León, México Sergio Zamudio

Investigador Independiente, México Melisa Zini

Instituto de Botánica del Nordeste, Argentina Susana Zuloaga

Universidad de Guadalajara, México 